\title{
Experimental and numerical study of the recirculation flow inside a liquid meniscus focused by air
}

\author{
A. M. Gañán-Calvo $\cdot$ C. Ferrera $\cdot$ M. Torregrosa $\cdot$ \\ M. A. Herrada $\cdot$ M. Marchand
}

Received: 5 November 2010/ Accepted: 21 January 2011/Published online: 6 February 2011

(C) Springer-Verlag 2011

\begin{abstract}
The internal motions inside a liquid meniscus in the so-called liquid cone-jet mode, which can occur upon stimulation by a coflowing gas sheath in flow focusing, are explored by both numerical simulation and experimental visualization. The results for low viscosity liquids show that, as in previous numerical simulations, a recirculating cell inside the meniscus appears when the injected liquid flow rate is reduced. Thus, as the flow rate is reduced not only the average residence time of particles in the meniscus becomes longer, but the appearance of a recirculation cell provides a natural platform for the efficient micro-mixing of different species before they are ejected through the issuing jet. The numerical results were confirmed with experimental visualization of the flow inside the meniscus using a dyed liquid. However, when the viscosity of the liquid is increased the recirculating cell disappears. In this case, viscous stresses organize the streamlines and direct the flow to the meniscus tip, which prevents the recirculating cell from being formed even for very small injected rate of liquid flow.
\end{abstract}

Keywords Cone-jet mode Flow focusing $\cdot$ Flow structure visualization $\cdot$ Micro-mixing $\cdot$ Recirculating cell

\footnotetext{
A. M. Gañán-Calvo $(\bowtie) \cdot$ M. Torregrosa · M. A. Herrada Departamento de Ingeniería Aeroespacial y Mecánica de Fluidos, Universidad de Sevilla, 41092 Sevilla, Spain e-mail: amgc@us.es

C. Ferrera

Departamento de Física Aplicada, Universidad de Extremadura, 10071 Cáceres, Spain
}

M. Marchand

Ecole Nationale Supérieure de Mécanique et d'Aérotechniques, Téléport 2-1 Avenue Clément Ader, BP 40109, 86961 Futuroscope, France

\section{Introduction}

The flow focusing technique has been a fast developing field in the last two decades, mainly because its relatively simple geometry allows fine control and high production rates of drops, bubbles, jets, and other micro- and nanometric fluid structures (Gañán-Calvo 1998; Gañán-Calvo and Gordillo 2001; Anna et al. 2003; Herrada et al. 2008). Typical configurations consist of coflowing setups, where an internal fluid stream, usually conducted through a capillary tube, is focused through a constriction by a surrounding flow of a second immiscible fluid. The external fluid pulls on the meniscus interface, inducing, depending on the conditions, the formation of blobs (dripping mode) or a liquid cone jet (jetting mode) (Herrada et al. 2008). The basic configuration can be slightly modified by adding a third or more flows to the system injected through extra concentric capillaries. This arrangement has been studied for the production of multicore microcapsules (MartínBanderas et al. 2005) or double emulsions (Utada et al. 2005), with immiscible fluids being those that are focused.

A recent study (Herrada et al. 2008) reported the appearance of a recirculation cell (RC) in the liquid meniscus of a liquid jet focused by gas, observed for certain conditions in numerical simulations. They noted that the formation of the RC appeared to be linked to high interface velocities induced by the gas sheath, which sets in motion a liquid flow toward the cusp of the meniscus in excess of the imposed flow rate. This flow in excess, unable to get issued through the jet, returns back following essentially the axis line. The recirculation flow seems to be analogous to the meridional motions observed inside Taylor cones for high liquids viscosities and electrical conductivities (Barrero et al. 1999). A similar feature has been experimentally observed in a two-dimensional 
liquid-liquid microfluidic flow focusing device (Anna and Mayer 2006). Although the study focused on the use of surfactant-mediated tip-streaming to synthesize droplets, a streamline pattern showed circulation of the inner focused flow along two symmetric vortices about the axis of the cone. A common feature of these flows with a cusp-like meniscus from whose tip a steady jet issues is the conspicuous presence of meridional viscous tangential stresses acting on the meniscus surface in the direction of the cusp. As seen from the tip of the meniscus, the cusp geometry in combination with those tangential stresses usually result in flow patterns strikingly compatible with the trailing end of viscous drops moving through viscous liquids, a classic kind of problem studied by salient authors (see Hadamard 1911; Rybczynski 1911; Clift et al. 1978 and cites therein). As seen from the top of the meniscus, the imposed flow rate coming from the feeding tube faces the excess back flow coming along the axis from the cusp, resulting in a flow pattern locally analogous to the leading edge of the mentioned viscous drops moving in viscous liquids. Consequently, the resulting global flow should contain a steady Hill's-like axisymmetric vortex (Herrada et al. 2008), a very efficient micromixing device. In this case, the internal flow is not separated from the surrounding flow by a free interface with surface tension but by a closed stream tube (in an ideally axisymmetric configuration) since both inner and outer liquids are essentially the same.

A great deal of study on microfluidic micromixing has been published in the literature, most of it motivated by the intrinsic difficulty of efficient mixing at the low Reynolds and Peclet numbers characteristic of most microfluidic flows. In particular, micromixing in droplets has been the subject of several works (see Song et al. 2006 and references therein), some of which demonstrated that good mixing can be attained by forcing the droplets to flow through undulated or labyrinth-like channels. Very recently, Liu et al. (2010) have proposed the generation of internal mixing inside hanging droplets by the action of an external gas stream. However, the possibility of using the capillary flow focusing technique with focused miscible fluids to enhance the interaction between the fluids within the liquid meniscus prior to the eventual discharge through the issuing micro-jet has not been studied previously. This approach may eventually lead to a one-step procedure in which highly efficient micromixing and subsequent massive nearly monodisperse micro-droplet generation can be attained. The flow is characterized by very different length and time scales in the capillary quasi-static meniscus or in the issuing micro-jet. This fact may open unprecedented avenues for the fine control of chemical reactions at the microscale, and to the efficient production of multicomponent or specifically designed microbeads, high quality powders or microstructured microfibers. For the purpose of this study, a few observations can be extracted from the published studies on flow focusing: (1) the fact that the RC observed through numerical simulations appears under certain conditions only and with different shapes depending on the case shows that this feature can be controlled; (2) as far as the authors know, there has been no reported experimental evidence for the $\mathrm{RC}$ inside the meniscus of an axisymmetric flow focusing device yet; (3) the manipulation of the recirculation motions inside the liquid meniscus is an interesting feature that could be applied to control the interaction between miscible fluids, and (4) a capillary flow focusing configuration using concentric capillaries to inject the miscible fluids inside the meniscus seems to be an appropriate configuration with which to study this feature, as it has been successfully used for other fluid combinations (immiscible fluids or different phases).

In this study, a capillary flow focusing device using gas as the focusing phase and different combinations of miscible fluids injected through concentric capillary tubes has been used to give a general picture of the possibilities of controlling the interaction between the miscible phases by means of manipulating the recirculation motion inside the liquid meniscus. The study is based on both numerical simulations and experimental observations, using quantitative analysis of the meniscus shape and qualitative visualization of the RC by means of tinted flows to validate the hypotheses in the numerical simulations. Aspects such as geometrical configurations, fluid viscosities, and flow rates were explored numerically and experimentally in this study to give a general idea of the possibilities of this configuration to control the RC. The aim is for this to be a starting point for further studies such as the parametrization and modeling of the phenomena involved, which fall beyond the scope of this study.

\section{Experimental procedure}

Figure 1 shows the experimental setup used in this study. Two miscible liquids were injected by two-independent infusion pumps (Harvard Apparatus, 11 Plus) through two concentric stainless steel capillary tubes (A), with outer diameters $D_{\text {lout }}=0.36 \mathrm{~mm}$ and $D_{\text {2out }}=1 \mathrm{~mm}$, and inner diameters $D_{1 \text { in }}=0.15 \mathrm{~mm}$ and $D_{2 \text { in }}=0.7 \mathrm{~mm}$, respectively (see Fig. 2). The concentricity of the tube outlets was manually achieved using appropriate mechanical tools. That concentricity was monitored with two cameras placed perpendicularly and equipped with high magnification objectives. In this study, the tube outlets were positioned in the same plane and aligned with a hole (B) of diameter $D_{0}$ drilled in a thin plate located at a distance $H_{0}$. The plate was firmly attached to a container (C), and the distance $H_{0}$ was set using three translation stages (D) to displace both 


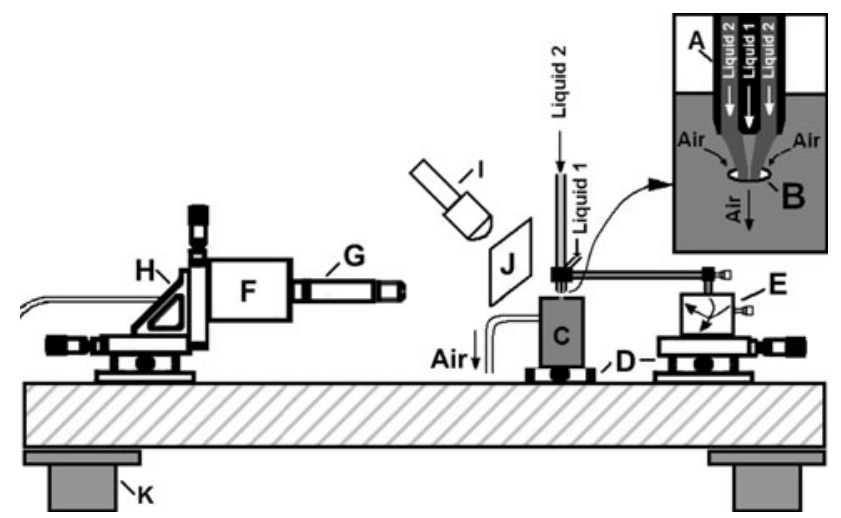

Fig. 1 Experimental setup: $(A)$ capillary tubes, $(B)$ hole in the plate, $(C)$ container, $(D)$ translation stages, $(E)$ dual-axis goniometer, $(F)$ high-speed video camera, $(G)$ objective, $(H)$ micrometric screw 3D-system, $(I)$ optical fiber, $(J)$ frosted diffuser, and $(K)$ optical table

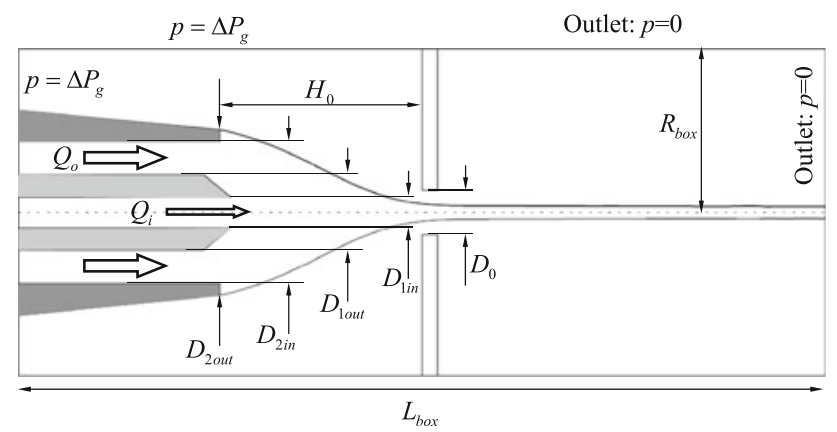

Fig. 2 Geometrical dimensions of the physical domain under consideration. Also shown, dimensions of the box and boundary conditions in the numerical simulations

tubes and container. The inclination of the tubes was controlled by means of a dual-axis goniometer (E). The container was connected to a vacuum pump (Telstar P-3), a pressure regulator, and a manometer. Once the setup was completed, the vacuum pump originated a pressure drop of $\Delta P_{g}$. This pressure drop created a meniscus at the tubes' exit, and pulled the injected liquids through the orifice (flow focusing technique). The flow structure in the liquid meniscus could be visualized by suitable choice of the liquids. This was achieved by injecting a transparent outer liquid and a colored or opaque inner liquid. A high-speed camera (Redlake MotionPro X4) (F) equipped with optical lenses $(\mathrm{G})$ was installed to take digital images of the liquids in the region between the tube exits and the orifice (Fig. 1). The camera was placed perpendicularly to the axis of the capillary tubes, and could be oriented with three micrometric screws $(\mathrm{H})$. The fluid configuration was illuminated by a cool white light beam at $45^{\circ}$ azimuth from the camera axis. The light was provided by an optical fiber
(I) connected to a light source (Schott KL2500 LCD). A frosted diffuser $(\mathrm{J})$ was positioned between the optical fiber and the capillary tubes to provide uniform light. All the equipment described was mounted on an optical table $(\mathrm{K})$.

In order to investigate in detail the onset of recirculation, the flow rate of the innermost capillary fluid (the colored liquid) was fixed at $Q_{i}=90 \mu \mathrm{L} \mathrm{h}^{-1}$ through all the experiments, and the outer capillary flow rate $Q_{\mathrm{o}}$ ranged from 20 to $150 \mathrm{~mL} \mathrm{~h}^{-1}$.

Figure 3 shows the evolution of the fluid structure as $Q_{\mathrm{o}}$ decreases for experiments carried out with distilled water and ink as outer and inner flows, respectively. For $Q_{\mathrm{o}}$ sufficiently high (Fig. 3a), the ink trajectory is almost straight, with the mixing with the surrounding liquid flow being negligible. As $Q_{\mathrm{o}}$ decreases, the ink trajectory deviates from the centreline (Fig. 3b, c). At a relatively well-defined threshold, the ink path unfolds into a recirculating pattern within the conical meniscus (Fig. 3d-f). The appearance of this structure not only dramatically increases the residence time of the ink flow in the focusing meniscus, but also generates a multiply folding toroidal structure (vortex ring) allowing rapid mixing. Indeed, both liquids are completely mixed before being pulled through the orifice when $Q_{\mathrm{o}}$ is below the aforementioned threshold (Fig. 3e, f).

On the other hand, the flow inside the meniscus becomes stationary if one fixes both $Q_{\mathrm{o}}$ and $Q_{\mathrm{i}}$. Figure 4 shows three experimental images recorded at $250 \mathrm{fps}$ for the same conditions. The images are almost identical independently of the time when they were taken. Therefore, while the experimental setup allows us to identify the parameter region for which a recirculating pattern appears, the authors cannot quantify the velocity field inside the meniscus. Experimental procedures combining micro-PIV with confocal microscopy have been proposed to visualize and quantify these internal flows in microfluidics (Chen et al. 1997; Ahn et al. 2008). However, the prior experience and results from numerical simulations of these micro-flows using Volume-of-Fluid (VOF) schemes (Herrada et al. 2008; Herrada and Gañán Calvo 2009) show that numerical experiments reproduce with remarkable fidelity and accuracy all the dynamical quantities involved in these physical phenomena. The experimental setup selected in this study (see Sect. 2) permits direct comparison between numerical simulations (Sect. 3) and experimental images (Sect. 4). Thus, the authors chose the combination of a visual observation (including a precise delimitation of the meniscus contour) under carefully controlled working conditions and the corresponding numerical simulation to gain the insight sought in this study. 

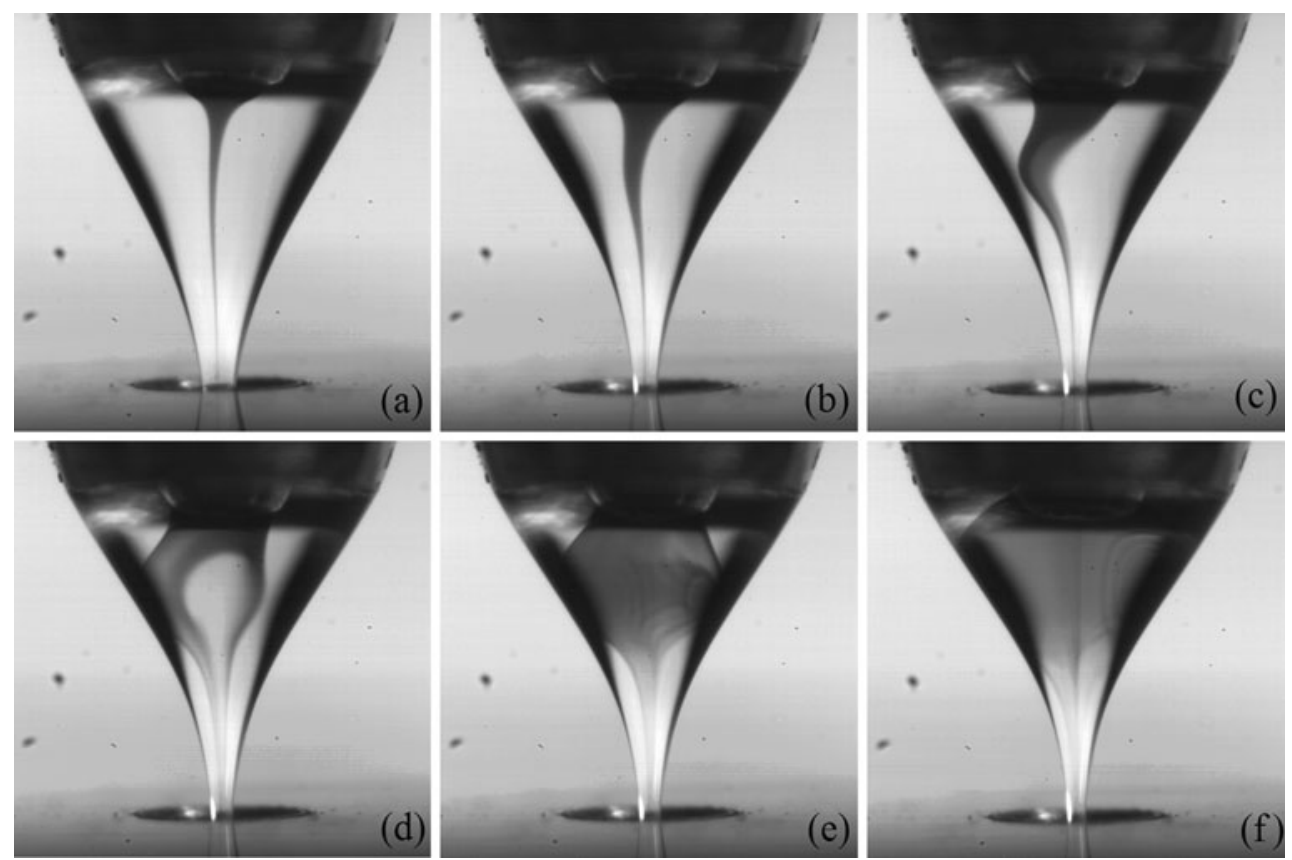

Fig. 3 Experimental images for $D_{0}=400 \mu \mathrm{m}, H_{0}=800 \mu \mathrm{m}, \Delta P_{g}=590 \mathrm{mbar}$, and six water flow rates $\left(\mathbf{a} Q_{o}=150 \mathrm{mLh}^{-1}\right.$, b $Q_{o}=75 \mathrm{~mL} \mathrm{~h}^{-1}$, c $Q_{o}=65 \mathrm{mLh}^{-1}$, d $Q_{o}=60 \mathrm{mLh}^{-1}$, e $Q_{o}=55 \mathrm{~mL} \mathrm{~h}^{-1}$, f $Q_{o}=40 \mathrm{~mL} \mathrm{~h}^{-1}$ )
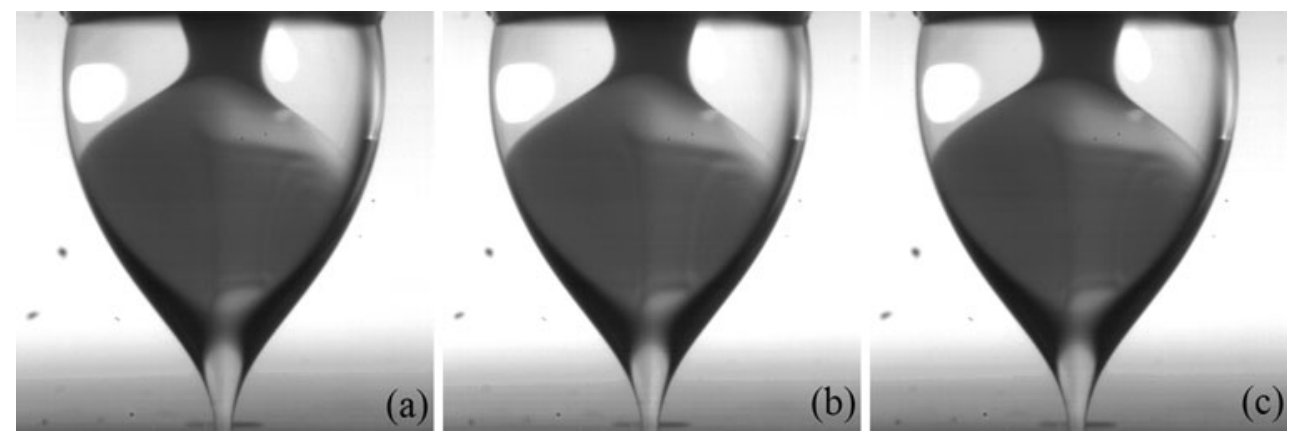

Fig. 4 Experimental images for $D_{0}=200 \mu \mathrm{m}, H_{0}=1000 \mu \mathrm{m}, \Delta P_{g}=610 \mathrm{mbar}, Q_{o}=35 \mathrm{mLh}^{-1}$ taken at three different times (a $t=0 \mathrm{~s}$, b $t=0.024 \mathrm{~s}, \mathbf{c} t=0.092 \mathrm{~s}$

\section{Numerical simulations}

The authors performed axisymmetric flow simulations for three geometrical configurations $\left(D_{0}=200 \mu \mathrm{m}, H_{0}=\right.$ $1000 \mu \mathrm{m} ; D_{0}=400 \mu \mathrm{m}, H_{0}=1000 \mu \mathrm{m} ;$ and $D_{0}=200 \mu \mathrm{m}$, $\left.H_{0}=750 \mu \mathrm{m}\right)$

The authors used a finite volume scheme provided by the commercial solver FLUENT v 6.3 (laminar unsteady) to resolve the mass continuity, conservation of momentum, and liquid volume fraction equations for the incompressible regime. The equations were discretized using a basic mesh, which usually consisted of 22,500 cells. The cells were quadrangles, mostly squares of $5 \mu \mathrm{m}$ in length. The basic mesh was refined to capture, in the absence of the liquid, the strong velocity gradients experienced by the gas flow in the nozzle region. Further mesh refinements were used in the interface region to avoid numerical diffusion of the interface when the liquid stream was supplied.

Tracking the interface between the liquid and focusing gas phases was accomplished by solving a continuity equation for the volume fraction of one of the phases from an explicit time-marching scheme. The time step was sufficiently small to insure that the global Courant number based on the time step, the mesh cell size, and the mean velocity in the cell was always less than unity. Regarding the spatial discretization of the equations, the third-order modified MUSCL scheme (Issa 1986) was used to obtain the face fluxes whenever a cell was completely immersed in a single phase. When the cell was near the interface, the Geo-Reconstruction algorithm was used. The pressure 
corrections were computed with the body forces weighted scheme, and the pressure-velocity coupling in a segregated solver was treated by the PISO method (Leer 1979).

Figure 2 depicts the integration domain and the boundary conditions imposed in the numerical simulations to faithfully represent the experimental set up. While the experiments were conducted in an unbounded domain, the numerical domain was a bounded box with $R_{\text {box }}=0.004 \mathrm{~m}$ and $L_{\text {box }}=0.002 \mathrm{~m}$ (see Fig. 2). At the gas inlet, the gauge pressure $\Delta P_{g}$ was prescribed, and the velocity direction was set normal to the boundary. Since the colored flow was injected through a relatively long (about $80 \mathrm{~mm}$ ) and very small bore capillary $\left(D_{1 \text { in }}=150 \mu \mathrm{m}\right)$ with a Reynolds number of about 0.14 , the velocity profile corresponding to the flow rate $Q_{\mathrm{i}}$ was assumed parabolic at the inner tube inlet. In contrast, a plug profile corresponding to the flow rate $Q_{\mathrm{o}}\left(Q_{o, \text { min }}=20 \mathrm{~mL} \mathrm{~h}^{-1}\right.$, with a Reynolds number of about 3.3 for water) was imposed at the outer inlet. Zero gauge pressure was set at the outlet, which was located far from the nozzle region $\left(R_{\text {box }}, L_{\text {box }} \gg D_{0}\right)$. The non-slip boundary condition was assumed at the solid walls. The contact angle at the capillary end was selected to allow the triple contact line to reach the outer capillary edge in the course of the simulations, as occurred in the experiments. The simulations were started from rest by injecting the fixed ink flow rate $Q_{\mathrm{i}}$ and a flow rate $Q_{\mathrm{o}}$ sufficiently high to obtain steady jetting; once the steady jetting regime was reached, $Q_{\mathrm{o}}$ was reduced progressively to different prescribed values until a steady state was reached, to investigate the non-linear steady flow pattern dependence on this parameter.

\section{Results}

Figure 5 shows the comparison between the experimental and the numerical results for $D_{0}=400 \mu \mathrm{m}$, $H_{0}=1000 \mu \mathrm{m}, \Delta P_{g}=590 \mathrm{mbar}$, and three values of $Q_{\mathrm{o}}$. Distilled water and ink were used as the outer and inner injected fluids, respectively. The experimental images were processed using a procedure described in Ferrera et al. (2006) to obtain the contour of the liquid-gas meniscus. The contour was superimposed on the streamlines obtained from the numerical simulations. The shape of the meniscus is remarkably well predicted by the numerical simulations for the three cases shown in the figure. The appearance of the recirculation flow calculated in the simulations agrees with the deviation in the trajectory and the eventual mixing of the ink observed in the experiments as $Q_{\mathrm{o}}$ decreases.

Similar results were obtained for other geometrical configurations. Figure 6 shows the comparison between the experimental and the numerical results for $D_{0}=200 \mu \mathrm{m}$, $H_{0}=1000 \mu \mathrm{m}, \Delta P_{g}=610 \mathrm{mbar}$, and three values of $Q_{\mathrm{o}}$. Once again, the shape of the meniscus is remarkably well predicted by the numerical simulations and the appearance of the recirculating flow observed in he simulations agrees with the deviation in the trajectory and the eventual mixing of the ink observed in the experiments.

The results shown in Figs. 5 and 6 demonstrate that a recirculating flow appears inside the water meniscus as the injected flow rate is reduced. The following results will show that the situation changes when higher viscosity liquids are used; indeed, that the viscosity of the liquid play a crucial role in the dynamics of the flow inside the meniscus is demonstrated by the experimental results obtained with $H_{0}=1000 \mu \mathrm{m}, \Delta P_{g}=610 \mathrm{mbar}$, a fixed flow rate $Q_{o}=30 \mathrm{~mL} \mathrm{~h}^{-1}$, and three water-glycerin mixtures, as Fig. $7 \mathrm{a}-\mathrm{c}$ shows. In all cases the surface tension variations were small, ranging from 52.3 to $55.8 \mathrm{mN} / \mathrm{m}$. It can be seen in the images how the recirculating flow completely disappears as the viscosity increases. This effect was repeated for a different geometrical configuration $\left(H_{0}=750 \mu \mathrm{m}\right)$ (see Fig. $7 \mathrm{~d}-\mathrm{f}$ ).

Interestingly, by decreasing the the injected flow rate $Q_{\mathrm{o}}$ when high viscosity liquids are used, the meniscus becomes unstable (the transition to a dripping regime is reached) before the recirculating cell appears. For example, Fig. 8 shows the comparison between the experimental and the numerical results for $D_{0}=200 \mu \mathrm{m}, H_{0}=750 \mu \mathrm{m}$, $\Delta P_{g}=610 \mathrm{mbar}, Q_{o}=20 \mathrm{~mL} \mathrm{~h}^{-1}$, and two liquids (viscosities: 5 and $12 \mathrm{cp}$ ). In both cases, the value of $Q_{\mathrm{o}}$ selected was close to the minimum value of the flow rate compatible with a stable meniscus. The numerical simulations allow one to visualize the changes in the topology of the flow inside the meniscus as the viscosity is increased: viscous stresses "flatten" the streamlines, and direct the flow to the meniscus tip, preventing the formation of a recirculating cell.

To rationalize the potential appearance of recirculation within the stable meniscus regime, one needs to introduce useful figures of merit. In this regard, one may compare the basic flow with characteristic velocity $Q_{o} / D_{0}^{2}$ with the surface velocity $v_{\mathrm{s}}$ induced by the outer gas stream. First, the thickness of the boundary layers at both the liquid $\left(\delta_{1}\right)$ and the gas side $\left(\delta_{\mathrm{g}}\right)$ can be estimated as

$$
\delta_{l} \sim \frac{D_{0}}{\left(\frac{\rho_{l} v_{\mathrm{s}} D_{0}}{\mu_{l}}\right)^{1 / 2}}, \quad \delta_{\mathrm{g}} \sim \frac{D_{0}}{\left(\frac{\rho_{\mathrm{g}} U_{\mathrm{g}} D_{0}}{\mu_{\mathrm{g}}}\right)^{1 / 2}}
$$

where $U_{\mathrm{g}}$ is the characteristic velocity of the gas at the vicinity of the discharge orifice, i.e., $U_{\mathrm{g}} \sim\left(\Delta P / \rho_{\mathrm{g}}\right)^{1 / 2}$. Here, $\rho$ and $\mu$ are the density and viscosity of the corresponding fluid and the subindexes 1 and $g$ refer to 

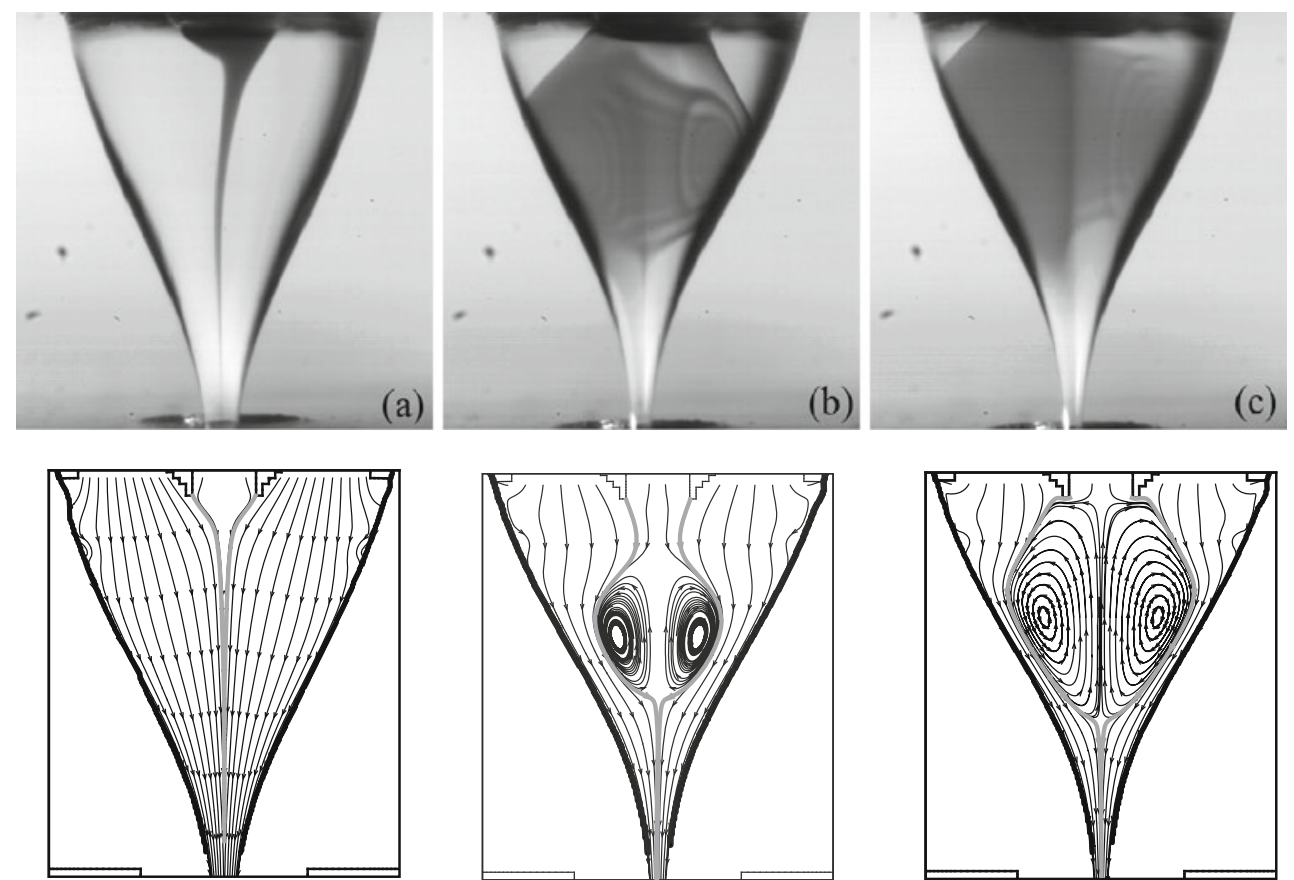

Fig. 5 Comparison between experimental images (top) and numerical results (bottom) for different flow rates $\left(\mathbf{a} Q_{o}=150 \mathrm{~mL} \mathrm{~h}^{-1}\right.$, b $Q_{o}=50 \mathrm{mLh}^{-1}$, c $Q_{o}=40 \mathrm{mLh}^{-1}$ ). The points in the lower images correspond to the contour detected in the top images, and the

solid lines are the numerical streamlines. The thick gray streamline divides both inner and outer focused fluids. Experiments and simulations were conducted with distilled water, $\Delta P_{g}=590 \mathrm{~m}$ bar, and $Q_{i}=90 \mu \mathrm{Lh}^{-1}\left(D_{0}=400 \mu \mathrm{m}, H_{0}=1000 \mu \mathrm{m}\right)$
Fig. 6 Comparison between experimental images (top) and numerical results (bottom) for different flow rates

(a $Q_{o}=100 \mathrm{~mL} \mathrm{~h}^{-1}$,

b $Q_{o}=40 \mathrm{~mL} \mathrm{~h}^{-1}$,

c $Q_{o}=30 \mathrm{~mL} \mathrm{~h}^{-1}$ ). The points in the lower images correspond to the contour detected in the top images, and the solid lines are the numerical streamlines. The thick gray streamline divides both inner and outer focused fluids. Experiments and simulations were conducted with distilled water,

$\Delta P_{g}=610 \mathrm{mbar}$, and $Q_{i}=$ $9 \mu \mathrm{Lh}^{-1}\left(D_{0}=200 \mu \mathrm{m}\right.$, $\left.H_{0}=1000 \mu \mathrm{m}\right)$
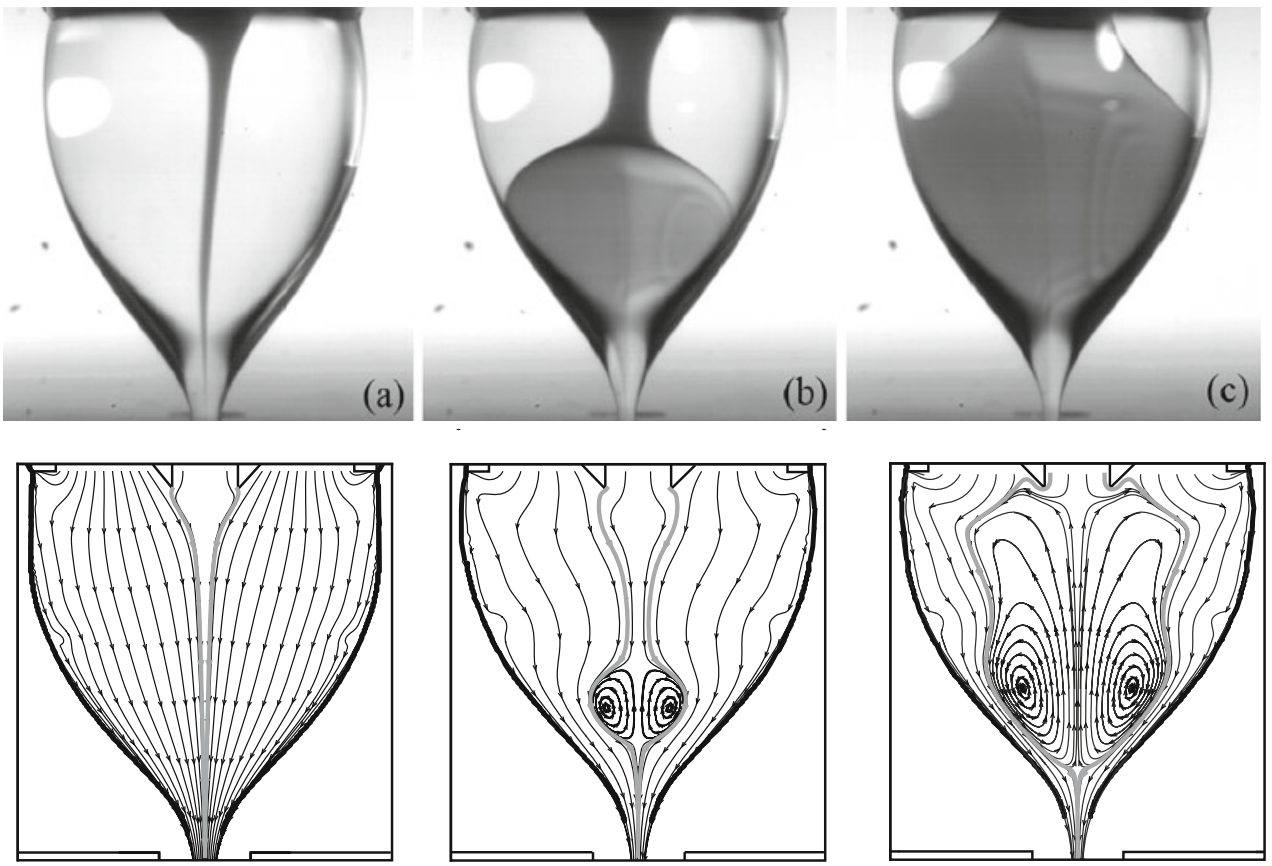

the liquid and the gas phase, respectively. In the above estimations, since $\rho_{\mathrm{g}} \ll \rho_{\mathrm{l}}$ and $\mu_{\mathrm{g}} \ll \mu_{\mathrm{l}}$, one naturally has $Q_{o} / D_{0}^{2} \ll v_{s} \ll U_{\mathrm{g}}$ as long as the diameter of the issued liquid jet $d_{\mathrm{j}} \sim\left(\frac{\rho_{1}}{\Delta P}\right)^{1 / 4} Q_{o}^{1 / 2}$ is small compared to $D_{0}$. The balance of tangential stresses at the surface of the meniscus demands 

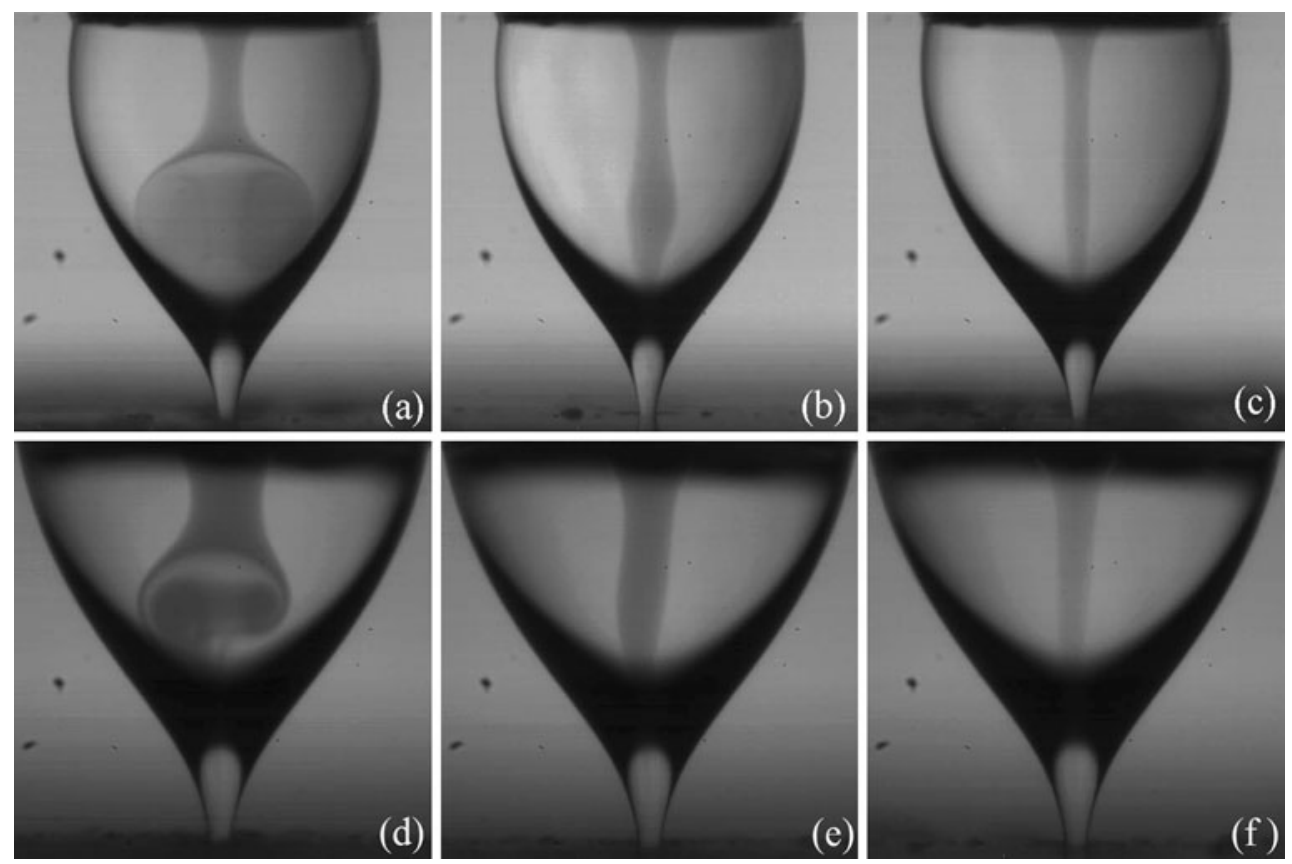

Fig. 7 Experimental images for $D_{0}=200 \mu \mathrm{m}, \Delta P_{g}=610 \mathrm{mbar}, Q_{0}=30 \mathrm{mLh}^{-1}$, two values of $H_{0}, H_{0}=1000 \mu \mathrm{m}(\mathbf{a}-\mathbf{c})$ and $H_{0}=750 \mu \mathrm{m}$ (d-f), and three water-glycerin mixtures (viscosities: a, d $5 \mathrm{cp}, \mathbf{b}, \mathbf{e} 7.09 \mathrm{cp}, \mathbf{c}, \mathbf{f} 12 \mathrm{cp}$ )
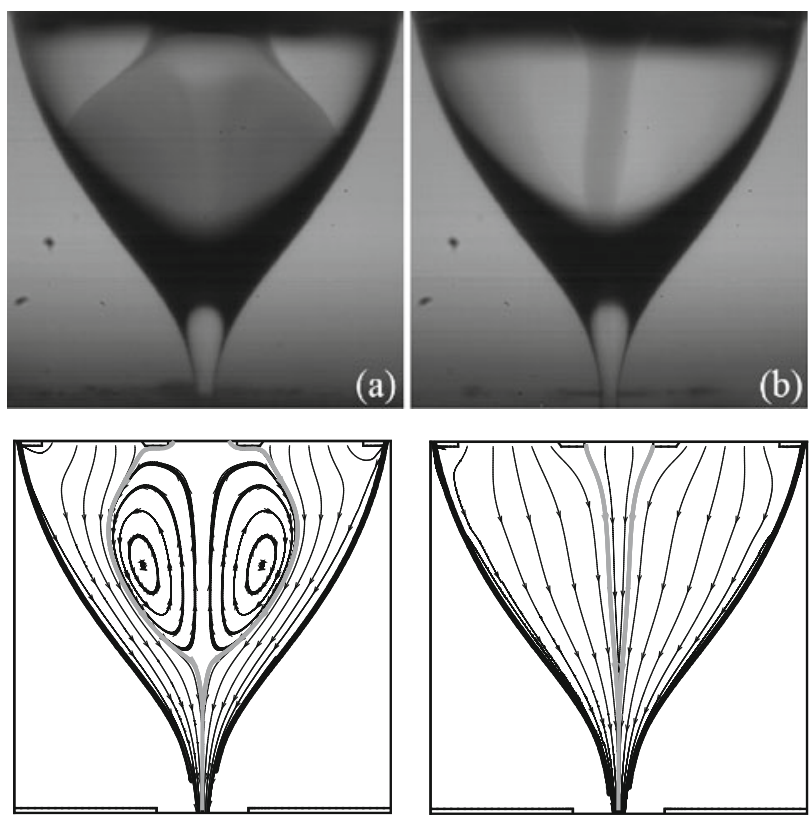

Fig. 8 Comparison between experimental images (top) and numerical results (bottom) for $Q_{o}=20 \mathrm{~mL} \mathrm{~h}^{-1}$, and two different waterglycerin mix liquids of viscosities: a $5 \mathrm{cp}$ and $\mathbf{b} 12 \mathrm{cp}$. The points in the lower images correspond to the detected contour of the top images, and the solid lines are the numerical streamlines. The thick gray streamline divides both inner and outer focused fluids. Experiments and simulations were conducted with $\Delta P_{g}=610 \mathrm{mbar}$ and $Q_{i}=90 \mu \mathrm{Lh}^{-1}$ $\mu_{\mathrm{g}} \frac{U_{\mathrm{g}}}{\delta_{\mathrm{g}}} \sim \mu_{l} \frac{v_{\mathrm{s}}}{\delta_{\mathrm{l}}}$,

which yields

$v_{\mathrm{s}} \sim U_{\mathrm{g}}\left(\frac{\rho_{\mathrm{g}} \mu_{\mathrm{g}}}{\rho_{1} \mu_{\mathrm{l}}}\right)^{1 / 3}$.

Secondly, if the Reynolds number $\operatorname{Re}_{\mathrm{s}}=\rho_{1} v_{\mathrm{s}} D_{0} / \mu_{1}$ is larger than unity, the order of magnitude of the velocity of the back-flow in the form of an axial liquid jet is the same as the surface velocity $v_{\mathrm{s}}$ by conservation of kinetic energy arguments: to this end, consider the classical problem of a liquid jet impinging perpendicularly on a flat plate, but in reverse direction. Therefore, two notable figures of merit should be (i) the Reynolds number of the base-flow driven by the imposed flow rate $Q_{\mathrm{o}}$, defined as:

$\operatorname{Re}_{\mathrm{D}}=\frac{\rho_{1} Q_{o}}{\mu_{1} D_{0}}$

and (ii) the Reynolds number $\operatorname{Re}_{\mathrm{s}}$ of the surface velocity $v_{\mathrm{s}}$. Now, since the interest is determined by the mixing properties of the resulting flow, we may introduce the ratio of the characteristic time associated to the recirculating flow, $t_{\mathrm{RC}} \sim D_{0} / v_{\mathrm{s}}$ to the characteristic diffusion time $t_{\mathrm{D}} \sim D_{0}^{2} / D_{\mathrm{BA}}$, where $D_{\mathrm{BA}}$ is the diffusion coefficient of species $\mathrm{B}$ in the liquid phase $\mathrm{A}$. The characteristic value $G_{\mathrm{D}}$ of the ratio $t_{\mathrm{RC}} / t_{\mathrm{D}}$ can be defined as: 
$t_{\mathrm{RC}} / t_{\mathrm{D}} \sim G_{\mathrm{D}}=\frac{D_{\mathrm{BA}} / v_{1}}{\operatorname{Re}_{\mathrm{s}}}=\left(\operatorname{Re}_{\mathrm{s}} \mathrm{Sc}\right)^{-1}$,

where $\mathrm{Sc}=v_{\mathrm{l}} / D_{\mathrm{BA}}$ is the Schmidt number. Naturally, the relevant figure of merit would be $G_{\mathrm{D}}$ instead of $\mathrm{Re}_{\mathrm{s}}$, but since in the case of liquid species $\mathrm{Sc}$ is usually of the order of $10^{3}$, unless sub-scales significantly smaller than $D_{\mathrm{o}}$ are developed (e.g., via the appearance of a recirculation cell), the molecular diffusion itself is unable to provide mixing. One can predict a drastic improvement in mixing by the use of the configuration described in this study. To show this, the authors have determined the flow rate at the onset of recirculation for different experiments, both real (from conditions shown in Figs. 3 and 7) and numerical (from Herrada et al. 2008), representing the corresponding values of $\mathrm{Re}_{\mathrm{s}}$ and $\mathrm{Re}_{\mathrm{D}}$ in Fig. 9. As long as $\mathrm{Re}_{\mathrm{s}}$ and $\mathrm{Sc}$ are both large, not only $t_{\mathrm{RC}}$ is small compared with $t_{\mathrm{D}}$, but also the back-flow motion driven by the surface velocity $v_{\mathrm{s}}$ overcomes the flow driven by $Q_{\mathrm{o}}$ and mixing becomes drastically enhanced by the development of recirculation (with characteristic cycling time $t_{\mathrm{RC}}$ ). Indeed, one can observe in Figs. 3 and 5 that the injected dyed liquid species does not mix at all and gets issued in the core of the emitted capillary jet in the absence of recirculation, while it becomes mixed as soon as recirculation develops. Therefore, good mixing and recirculation are simultaneously represented by the parametrical plane described by the parameters (figures of merit) $\mathrm{Re}_{\mathrm{s}}$ and $\mathrm{Re}_{\mathrm{D}}$.

Noteworthy, the asymptotic trend shown in Fig. 9 as $\operatorname{Re}_{\mathrm{s}} \sim \operatorname{Re}_{\mathrm{D}}$ (specifically, $\operatorname{Re}_{\mathrm{S}} / \operatorname{Re}_{\mathrm{D}} \simeq 27$ ) points to the following intriguing result

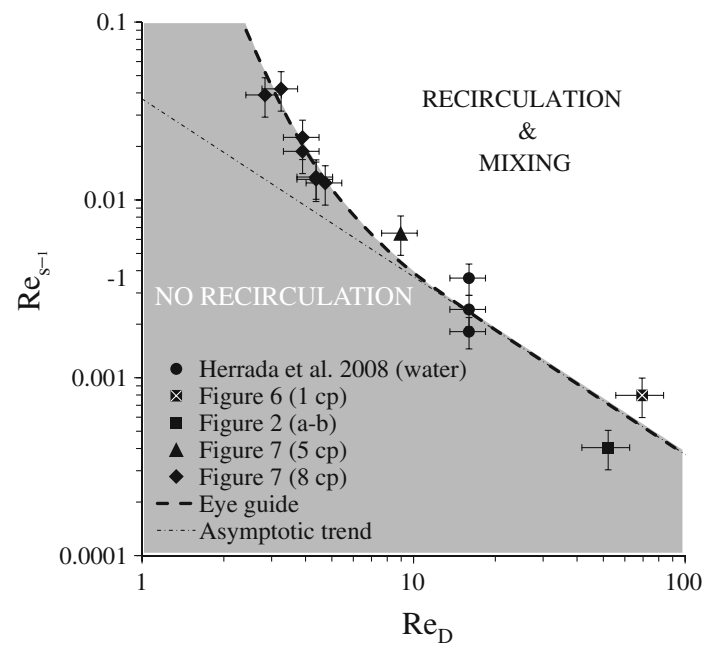

Fig. 9 Values of $\operatorname{Re}_{\mathrm{s}}$ versus $\mathrm{Re}_{\mathrm{D}}$ at the onset of recirculation for several experiments and numerical (Herrada et al. 2008) simulations. The corresponding subdomain of recirculation and mixing is marked in gray. Data shown with error bars of $20 \%$
$\operatorname{Re}_{\mathrm{s}} / \operatorname{Re}_{\mathrm{D}} \simeq 27 \Longrightarrow \frac{\rho_{\mathrm{g}} Q_{\mathrm{g}}}{\rho_{1} Q_{o}} \simeq \frac{\dot{m}_{\mathrm{g}}}{\dot{m}_{1}} \simeq 27\left(\frac{\rho_{\mathrm{g}}^{2} \mu_{\mathrm{l}}}{\rho_{1}^{2} \mu_{\mathrm{g}}}\right)^{1 / 3}$,

where $\dot{m}_{\mathrm{g}}$ and $\dot{m}_{1}$ are the mass flows of the gas and the liquid through the discharge orifice. Now, representing in Fig. 10 the values of $\frac{\dot{m}_{g}}{\dot{m}_{1}}$ versus $\left(\frac{\rho_{g}^{2} \mu_{1}}{\rho_{1}^{2} \mu_{\mathrm{g}}}\right)^{1 / 3}$ one obtains that, indeed, the ratio of the mass flows is close to unity at the onset of recirculation (note that the experimental values of $27 \times\left(\frac{\rho_{g}^{2} \mu_{\mathrm{g}}}{\rho_{1}^{2} \mu_{\mathrm{g}}}\right)^{1 / 3}$ are of the order unity as well!). Recirculation appears for $\dot{m}_{\mathrm{g}} / \dot{m}_{1}>1$. This highly reproducible result has been observed as long as $\mathrm{Re}_{\mathrm{s}}$ is larger than unity (observable in the experiments with liquid viscosities ranging from 1 to $12 \mathrm{cp}$ ), and points to a physically sound global argument outlined in the following.

First, as demonstrated by the observations given above, the transfer of momentum from the gas to the liquid phase is so effective that the motion of both gas and liquid species induced by $\Delta P_{\mathrm{g}}$ involve comparable kinetic energies. Indeed, at distances upstream of the discharge orifice of the order of a few diameters $D_{0}$ one has

$\rho_{1} v_{\mathrm{s}}^{2} \sim \rho_{\mathrm{g}} U_{\mathrm{g}}^{2}\left(\frac{\rho_{1} \mu_{\mathrm{g}}^{2}}{\rho_{\mathrm{g}} \mu_{1}^{2}}\right)^{1 / 3}$,

where $\left(\frac{\rho_{1} \mu_{g}^{2}}{\rho_{\mathrm{g}} \mu_{1}^{2}}\right)^{1 / 3} \sim \mathrm{O}(1)$ for the liquids used. Thus, since said kinetic energies of both phases should be conserved down to the cross section of the discharge orifice, if the imposed flow rate $Q_{\mathrm{o}}$ is reduced such that $\dot{m}_{\mathrm{g}}$ dominates

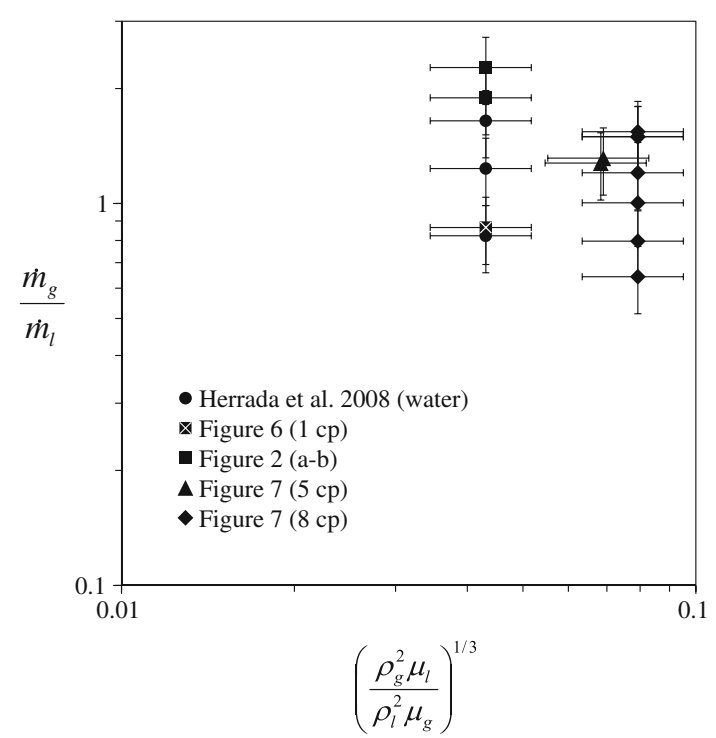

Fig. 10 The mass flow ratio $\dot{m}_{\mathrm{g}}$ and $\dot{m}_{\mathrm{l}}$ versus $\left(\frac{\rho_{\mathrm{g}}^{2} \mu_{\mathrm{l}}}{\rho_{1}^{2} \mu_{\mathrm{g}}}\right)^{1 / 3}$ at the onset of recirculation. Data shown with error bars of $20 \%$ 
over $\dot{m}_{1}$, this immediately induces a restriction on the liquid flow (whose velocity is governed by the gas flow imposed by $\Delta P_{\mathrm{g}}$, not by $Q_{\mathrm{o}}$ !) as if a solid object or diaphragm would have been interposed at the orifice, which provokes the back-flow of part of the incoming liquid stream. In addition, study is required to gain further insight in the implications of this intriguing result in the field of flow focusing. Noteworthy, at first approach the distance $H_{0}$ is absent from the above reasoning and deductions, consistently with the argued dominance of the scale $D_{0}$ on the global dynamics of the system.

\section{Conclusions}

Experimental visualization of the recirculation flow inside the liquid meniscus of an axisymmetric liquid cone jet focused by air was made possible for the first time using the experimental setup described in this study for different liquid flow rates, viscosities, and geometrical configurations.

Numerical simulations were also executed in parallel showing good agreement with the experimental results for the different cases studied, not only between numerical and experimental meniscus shapes (detected from experimental images using the technique mentioned), but also for the recirculation flow present inside the meniscus. This recirculation flow exhibited a remarkable steadiness and robustness under fixed, controlled operating conditions. Further investigation to study the dynamical response of the system to imposed disturbances (e.g., periodic oscillations of the feeded flow rate or the imposed gas pressure) may very well reveal a vast wealth of dynamical responses, mixing enhancement and the like.

Based on the numerical and experimental results, the parameters studied allow control of the recirculation motions inside the RC. The relevant figures of merit to map the parametrical region where recirculation is present have been given, and that region is described in a twodimensional plane. The authors show that for low viscosity liquids, the injected liquid flow rate $Q_{\mathrm{o}}$ is a key parameter to manipulate the recirculation flow. Once the RC appears, it is possible to increase its size by decreasing the liquid flow rate $Q_{\mathrm{o}}$, until the system reaches the dripping mode. The results showed that getting recirculation motion within the meniscus for large viscosity liquids is not possible. When $Q_{\mathrm{o}}$ is reduced, dripping always takes place before the recirculation flow appears inside the liquid meniscus. Different geometrical configurations gave similar results. An intriguing observation points to the fact that at the onset of recirculation, the ratio of liquid and mass flow rates is close to unity.
For $\frac{\dot{m}_{\mathrm{g}}}{\dot{m}_{\mathrm{l}}}>1$, recirculation appears. As a general feature, the possibility of controlling the interface of interaction between miscible liquids focused by air makes the concentric capillary flow focusing technique a potential platform for such applications as micro-mixing and micro-reactions.

Acknowledgments This research was supported by the Ministerio de Educación y Ciencia (Spain) through Grant No. DPI2007-63559. Partial support from the Junta de Extremadura and the Junta de Andalucía through Grants Nos. GRU07003 and P08-TEP-04128, respectively, are also acknowledged. The authors wish to acknowledge nice suggestions from the referees as well.

\section{References}

Ahn YC, Jung W, Chen Z (2008) Optical sectioning for microfluidics: secondary flow and mixing in a meandering microchannel. Lab Chip 8:125-133

Anna SL, Mayer HC (2006) Microscale tipstreaming in a microfluidic flow focusing device. Phys Fluids 18:121,512

Anna SL, Bontoux N, Stone HA (2003) Formation of dispersions using flow focusing in microchannels. Appl Phys Lett 82(3): 364-366

Barrero A, Gañán-Calvo A, Dávila J, Palacios A, Gómez-González E (1999) The role of the electrical conductivity and viscosity on the motions inside Taylor cones. J Electrost 47:13-26

Chen Z, Milner T, Dave D, Nelson J (1997) Optical doppler tomographic imaging of fluid flow velocity in highly scattering media. Optics Lett 22:64-66

Clift R, Grace JR, Weber ME (1978) Bubbles, drops and particles. Academic Press, New York

Ferrera C, Cabezas MG, Montanero JM (2006) An experimental analysis of the linear vibration of axisymmetric liquid bridges. Phys Fluids 18:082105

Gañán-Calvo AM (1998) Generation of steady liquid microthreads and micron-sized monodisperse sprays in gas streams. Phys Rev Lett 80(2):285-288

Gañán-Calvo AM, Gordillo JM (2001) Perfectly monodisperse microbubbling by capillary flow focusing. Phys Rev Lett $87: 274501$

Hadamard JS (1911) Mouvement permanent lent d'une sphere liquide et visqueuse dans un liquide visqueux. C R Acad Sci 152:17351738

Herrada MA, Gañán-Calvo AM (2009) Swirl flow focusing: a novel procedure for the massive production of monodisperse microbubbles. Phys Fluids 21:042003

Herrada MA, Gañán-Calvo AM, Ojeda-Monge A, Bluth B, RiescoChueca P (2008) Liquid flow focused by a gas: jetting, dripping, and recirculation. Phys Rev E 78:036323

Issa RI (1986) Solution of implicitly discretized fluid flow equations by operator splitting. J Comput Phys 62:40-65

Leer BV (1979) Toward the ultimate conservative difference scheme. iv. a second order sequel to Godunov's method. J Comput Phys 32:101-136

Liu Z, Huang Y, Jin Y, Cheng Y (2010) Mixing intensification by chaotic advection inside droplets for controlled nanoparticle preparation. Microfluid Nanofluid 9:773-783

Martín-Banderas L, Flores-Mosquera M, Riesco-Chueca P, Rodríguez-Gil A, Cebolla Á, Chávez S, Gañán-Calvo AM (2005) Flow focusing: a versatile technology to produce size-controlled and specific-morphology microparticles. Small 1(7):688-692 
Rybczynski W (1911) ber die fortschreitende bewegung einer flnssigen kugel in einem zShen medium. Bull Int Acad Pol Sci Lett, Cl Sci Math Nat, Ser A 40-46:1735-1738

Song H, Chen DL, Ismagilov RF (2006) Reactions in droplets in microfluidic channels. Angew Chem Int Ed 45:7336-7356
Utada AS, Lorenceau E, Link DR, Kaplan PD, Stone HA, Weitz DA (2005) Monodisperse double emulsions generated from a microcapillary device. Science 308:537-541 